\title{
Combined Adomian Decomposition Method with Integral Transform
}

\author{
Betty Subartini $^{1, *}$, Ira Sumiati ${ }^{2}$, Sukono $^{1}$, Riaman $^{1}$, Ibrahim Mohammed Sulaiman ${ }^{3}$ \\ ${ }^{1}$ Department of Mathematics, Faculty of Mathematics and Natural Sciences, Universitas Padjadjaran, Jl. Raya Bandung Sumedang \\ KM.21, Hegarmanah, Jatinangor, Sumedang Regency, West Java 45363, Indonesia \\ ${ }^{2}$ Faculty of Mathematics and Natural Sciences, Universitas Padjadjaran, Jl. Raya Bandung Sumedang KM.21, Hegarmanah, \\ Jatinangor, Sumedang Regency, West Java 45363, Indonesia \\ ${ }^{3}$ Department of Mathematics and Statistics, School of Quantitative Sciences, Universiti Utara Malaysia, Sintok, Malaysia
}

Received September 2, 2021; Revised October 28, 2021; Accepted November 11, 2021

\section{Cite This Paper in the following Citation Styles}

(a): [1] Betty Subartini, Ira Sumiati, Sukono, Riaman, Ibrahim Mohammed Sulaiman , "Combined Adomian Decomposition Method with Integral Transform," Mathematics and Statistics, Vol. 9, No. 6, pp. 976 - 983, 2021. DOI: 10.13189/ms.2021.090613.

(b): Betty Subartini, Ira Sumiati, Sukono, Riaman, Ibrahim Mohammed Sulaiman (2021). Combined Adomian Decomposition Method with Integral Transform. Mathematics and Statistics, 9(6), 976 - 983. DOI: 10.13189/ms.2021.090613.

Copyright $\bigcirc 2021$ by authors, all rights reserved. Authors agree that this article remains permanently open access under the terms of the Creative Commons Attribution License 4.0 International License

\begin{abstract}
At present, three numerical solution methods have mainly been used to solve fractional-order chaotic systems in the literature: frequency domain approximation, predictor-corrector approach and Adomian decomposition method (ADM). Based on the literature, ADM is capable of dealing with linear and nonlinear problems in a time domain. Also, the Adomian decomposition method (ADM) is among the efficient approaches for solving linear and non-linear equations. Numerical solution method is one of the critical problems in theoretical research and in the applications of fractional-order systems. The solution is decomposed into an infinite series and the integral transformation to a differential equation is implemented in this work. Furthermore, the solution can be thought of as an infinite series that converges to an exact solution. The aim of this study is to combine the Adomian decomposition approach with a different integral transformation, including Laplace, Sumudu, Natural, Elzaki, Mohand, and Kashuri-Fundo. The study's key finding is that employing the combined method to solve fractional ordinary differential equations yields good results. The main contribution of our study shows that the combined numerical methods considered produce excellent numerical performance for solving fractional ordinary differential equations. Therefore, the proposed combined method has practical implications in solving fractional order differential equations in science
\end{abstract}

and social sciences, such as finding analytical and numerical solutions for secure communication system, biological system, financial risk models, physics phenomenon, neuron models and engineering application.

Keywords Adomian Decomposition Method, Natural Transform, Sumudu Transform, Laplace Transform, Elzaki Transform, Mohand Transform, Khasuri-Fundo Transform

Mathematics Subject Classification: 44A11; 65R10

\section{Introduction}

The ADM has many applications in science and technology [1]-[5], such as biological population model [6], SEIR epidemic models [7], arm robot [8], mobile robot navigation [9], financial risk chaotic system [10], dissipative magnetic Jeffrey biofluid [11], two-disk dynamo system [12], communication system based on Internet of Thing (IoT) [13], electronic circuit [14], electrostatic micro-actuators system [15], Simulated Rocket Motor [16] and field programmable gate array [17].

Some literature about Laplace transform and Adomian decomposition methods can be seen in [18]-[20]. Yindoula et al. [18] proposed the convection 
diffusion-dissipation equation using ADM and Laplace transform method. They show that the approximate solution of the equation has an exact solution. Zhen and Song [19] studied synchronization of FitzHugh-Nagumo neurons model using Laplace Transform and the ADM. They show that the Adomian decomposition approach yielded an exact analytical result for the two FitzHugh-Nagumo neurons models. Chen and Liu [20] solved a HIV infection model of CD4+ T Cells via Padé approximation process, ADM and Laplace transform method. They found that the Padé approximation and Laplace transform for obtaining the solutions of ADM is the most preferred approach for solving HIV infection model. Doğan [21] presented combined Laplace Transform and ADM for solution of the ordinary differential equations (ODEs). They found that the Combined Adomian decomposition- Laplace transform can be applied in the linear and non-linear systems of ODEs.

The Sumudu Decomposition Method has very important applications in mathematical fields, such as fractional Bratu-type differential equations [22], Volterra integrodifferential equations [23], Klein-Gordon equations [24], Space-Fractional Telegraph Equations [25], fractional Harry Dym equation [26], Riccati equation of variable fractional order [27] and Conformable Fractional Fitzhugh-Nagumo Model [28].

Previous studies about the Adomian-Natural decomposition approach and Elzaki decomposition method can be seen in [29]-[32]. Veeresha et al. [29] presented natural decomposition technique for solving fractional forced KdV equation. Khan et al. [30] proposed natural-transformation decomposition approach for fractional-order hyperbolic telegraph equation. Shah et al [31] analyzed fractional-order diffusion equations' transform decomposition method. Nuruddeen [32] solved linear and nonlinear Schrodinger using Elzaki decomposition method. Varsoliwala and Singh [33] studied model of atmospheric internal waves phenomenon using Elzaki decomposition method. Khan et al. [34] applied the Elzaki transform decomposition method and Caputo operator to solve the Navier-Stokes equations. In addition, the study of Mohand decomposition method and Kashuri-Fundo decomposition method can be seen in [35]-[38].

Based on the background study of previous research, this paper presents review the combination of Adomian decomposition methods with other integral transforms, such as Sumudu, Laplace, Natural, Elzaki, Mohand, and Kashuri-Fundo.

\section{Basic Theory}

\subsection{Integral Transform and Fractional}

This section describes the basic theories related to
Laplace, Sumudu, Natural, Elzaki, Mohand, and Kashuri-Fundo transforms.

Definition 2.1 [39]. Let $\boldsymbol{f}$ be a real or complex function of the value $\boldsymbol{t}>\mathbf{0}$ also, let $\boldsymbol{s}$ be a real or complex coefficient. Then, the Laplace transformation is defined as

$$
\mathcal{L}[\boldsymbol{f}(t)]=F(s)=\int_{0}^{\infty} e^{-s t} f(t) d t .
$$

Furthermore, $\mathcal{L}^{-1}[F(s)]=f(t), t \geq 0$ is the inverse of the Laplace transform. If $\alpha$ is a fractional number (FN), then

Definition 2.2 [40]. The definition of Laplace transformation of the Caputo fractional derivative (CFD) is given as

$$
\begin{gathered}
\mathcal{L}\left[D_{t}^{\alpha} f(t)\right]=s^{\alpha} F(s)-\sum_{k=0}^{n-1} s^{\alpha-k-1} f^{(k)}(0), n-1<\alpha \\
\leq n .
\end{gathered}
$$

Definition 2.3 [41]. Given a set of functions

$$
\begin{gathered}
\boldsymbol{S}=\left\{\boldsymbol{f}(\boldsymbol{t}): \exists \boldsymbol{M}, \boldsymbol{\tau}_{1}, \boldsymbol{\tau}_{2}>\mathbf{0},|\boldsymbol{f}(\boldsymbol{t})|<\boldsymbol{M} \boldsymbol{e}^{\frac{|t|}{\tau_{j}}, t}\right. \\
\left.\in(-1)^{j} \times[0, \infty)\right\},
\end{gathered}
$$

Sumudu transform is defined as

$$
\delta[f(t)]=G(u)=\int_{0}^{\infty} e^{-t} f(u t) d t, t \geq 0,-\tau_{1}<u<\tau_{2} .
$$

Furthermore, Sumudu transform's inverse is denoted as $\mathcal{S}^{-1}[G(u)]=f(t)$, for all $t \geq 0$. Suppose the parameter $\alpha$ represents FN, then

$$
\boldsymbol{s}\left[\boldsymbol{t}^{\alpha}\right]=\Gamma(\alpha+\mathbf{1}) \boldsymbol{u}^{\alpha} .
$$

Definition 2.4 [42]. The Sumudu transform of CFD is expressed as

$$
\mathcal{S}\left[D_{t}^{\alpha} f(t)\right]=\frac{G(u)}{u^{\alpha}}-\sum_{k=0}^{n-1} \frac{f^{(k)}(0)}{u^{\alpha-k}}, n-1<\alpha \leq n .
$$

Definition 2. 5 [43]. Given a set of functions

$$
\begin{gathered}
S=\left\{f(t): \exists M, \tau_{1}, \tau_{2}>0,|f(t)|<M e^{\frac{|t|}{\tau_{j}}, t}\right. \\
\left.\in(-1)^{j} \times[0, \infty)\right\},
\end{gathered}
$$

Natural transform is defined as

$$
\begin{gathered}
\mathcal{N}[f(t)]=H(s, u)=\int_{0}^{\infty} e^{-s t} f(u t) d t, t \geq 0, s>0,-\tau_{1} \\
<u<\tau_{2} .
\end{gathered}
$$

Furthermore, the inverse of the Natural transform is denoted as $\mathcal{N}^{-1}[\mathrm{H}(\mathrm{s}, \mathrm{u})]=\mathrm{f}(\mathrm{t}), \mathrm{t} \geq 0$. If $\alpha$ is a $\mathrm{FN}$, 
then

$$
\mathcal{N}\left[t^{\alpha}\right]=\Gamma(\alpha+1) \frac{u^{\alpha}}{s^{\alpha+1}} .
$$

Definition 2.6 [29]. The natural transform of the CFD is expressed as

$$
\begin{aligned}
\mathcal{N}\left[D_{t}^{\alpha} f(t)\right]= & \frac{s^{\alpha} H(s, u)}{u^{\alpha}}-\sum_{k=0}^{n-1} \frac{s^{\alpha-k-1}}{u^{\alpha-k}} f^{(k)}(0), n-1 \\
& <\alpha \leq n .
\end{aligned}
$$

Definition 2.7 [44]. Given a set of functions

$$
\begin{gathered}
\boldsymbol{S}=\left\{\boldsymbol{f}(\boldsymbol{t}): \exists M, \boldsymbol{\tau}_{1}, \boldsymbol{\tau}_{2}>\mathbf{0},|\boldsymbol{f}(\boldsymbol{t})|<M \boldsymbol{e}^{\frac{|t|}{\tau_{j}}, t}\right. \\
\left.\in(-1)^{j} \times[0, \infty)\right\},
\end{gathered}
$$

Elzaki transform is defined as

$\mathcal{E}[f(t)]=T(v)=v \int_{0}^{\infty} e^{-\frac{t}{v}} f(t) d t, t \geq 0,-\tau_{1}<v<\tau_{2}$.

Furthermore, Elzaki transform inverse is represented by $\mathcal{E}^{-1}[\mathrm{~T}(\mathrm{v})]=\mathrm{f}(\mathrm{t}), \mathrm{t} \geq 0$. If $\alpha$ is a FN, then

Definition 2.8 [44]. Elzaki transform of CFD is given as

$\varepsilon\left[D_{t}^{\alpha} f(t)\right]=\frac{T(v)}{v^{\alpha}}-\sum_{k=0}^{n-1} v^{k-\alpha+2} f^{(k)}(0), n-1<\alpha \leq n$.

Definition 2.9 [45]. Given a set of functions

$S=\left\{f(t): \exists M, \tau_{1}, \tau_{2}>0,|f(t)|<M e^{\frac{|t|}{\tau_{j}}}, t \in(-1)^{j} \times[0, \infty)\right\}$,

Mohand transform is defined as

$$
\begin{aligned}
\mathcal{M}[f(t)]=R(v) & =v^{2} \int_{0}^{\infty} e^{-v t} f(t) d t, t \geq 0,-\tau_{1}<v \\
& <\tau_{2} .
\end{aligned}
$$

Furthermore, Mohand transform inverse is represented by $\mathcal{M}^{-1}[\mathrm{R}(\mathrm{v})]=\mathrm{f}(\mathrm{t}), \mathrm{t} \geq 0$. If $\alpha$ is a FN, then

$$
\mathcal{M}\left[t^{\alpha}\right]=\frac{\Gamma(\alpha+1)}{v^{\alpha-1}} .
$$

Definition 2.10 [45]. The Mohand transform of CFD is given as

$$
\begin{gathered}
\mathcal{M}\left[D_{t}^{\alpha} f(t)\right]=v^{\alpha} R(v)-\sum_{k=0}^{n-1} v^{\alpha-k+1} f^{(k)}(0), n-1<\alpha \\
\leq n .
\end{gathered}
$$

Definition 2.11 [37]. Given a set of functions

$U=\left\{f(t): \exists M, \tau_{1}, \tau_{2}>0,|f(t)|<M e^{\frac{|t|}{\tau_{j}^{2}}}, t \in(-1)^{j} \times[0, \infty)\right\}$,

Kashuri-Fundo transform is expressed as

$$
\begin{gathered}
\mathcal{K}[f(t)]=W \int_{0}^{\infty} e^{-\frac{t}{v^{2}}} f(t) d t, t \geq 0,-\tau_{1}<u<\tau_{2} \cdot(v) \\
=\frac{1}{v}
\end{gathered}
$$

Furthermore, Mohand transform inverse is defined as $\mathcal{K}^{-1}[\mathrm{~W}(\mathrm{v})]=\mathrm{f}(\mathrm{t}), \mathrm{t} \geq 0$. If $\alpha$ is a FN, then

$$
\mathcal{K}\left[t^{\alpha}\right]=\Gamma(\alpha+1) v^{2 \alpha+1} .
$$

Definition 2.12 [37]. The Kashuri-Fundo transformation of CFD is given as

$$
\mathcal{K}\left[D_{t}^{\alpha} f(t)\right]=\frac{W(v)}{v^{2 \alpha}}-\sum_{k=0}^{n-1} \frac{f^{(k)}(0)}{v^{2(\alpha-k)-1}}, n-1<\alpha \leq n .
$$

\subsection{Adomian Decomposition Method}

The following discussion is about the basic concepts of the ADM

Given the following equation

$$
L y+N y+P y=g,
$$

where $\mathrm{L}$ represents the linear operator inverse, $\mathrm{N}$ is the nonlinear operator, and $\mathrm{P}$ denotes the remaining linear part. Equation (7) can be rewritten with Ly as the subject

$$
\boldsymbol{L y}=\boldsymbol{g}-\boldsymbol{N} \boldsymbol{y}-\boldsymbol{P} \boldsymbol{y} \text {. }
$$

Since $\mathrm{L}$ is inverse, the inverse operator of $\mathrm{L}$ is defined as $L^{-1}$. So that if both sides of equation (8) are inverted by $\mathrm{L}^{-1}$, we get

$$
y=\phi+L^{-1} g-L^{-1} N y-L^{-1} P y .
$$

The ADM supposes that $\mathrm{y}$ should be disintegrated into an infinite series

$$
y=\sum_{n=0}^{\infty} y_{n}=y_{0}+y_{1}+y_{2}+\cdots,
$$

where $\mathrm{y}_{\mathrm{n}}$ can be determined iteratively. ADM also supports the decomposition of nonlinear operator $\mathrm{Ny}$ into an infinite series of polynomial form:

$$
N y=\sum_{n=0}^{\infty} A_{n},
$$

where the Adomian polynomial (AP) is defined by $A_{n}=A_{n}\left(y_{0}, y_{1}, y_{2}, \ldots, y_{n}\right)$

$$
\begin{gathered}
A_{n}\left(y_{0}, y_{1}, y_{2}, \ldots, y_{n}\right)=\frac{1}{n !} \frac{d^{n}}{d \lambda^{n}}\left[N\left(\sum_{k=0}^{n} \lambda^{k} y_{k}\right)\right]_{\lambda=0}, n \\
=0,1,2, \ldots
\end{gathered}
$$

and the parameter is denoted by $\lambda . A_{n}$ is expressed as

$$
\begin{gathered}
A_{0}=\frac{1}{0 !} \frac{d^{0}}{d \lambda^{0}}\left[N\left(\sum_{k=0}^{0} \lambda^{k} y_{k}\right)\right]_{\lambda=0}=N\left(y_{0}\right), \\
A_{1}=\frac{1}{1 !} \frac{d^{1}}{d \lambda^{1}}\left[N\left(\sum_{k=0}^{1} \lambda^{k} y_{k}\right)\right]_{\lambda=0}=y_{1} N^{\prime}\left(y_{0}\right),
\end{gathered}
$$




$$
\begin{aligned}
A_{2}= & \frac{1}{2 !} \frac{d^{2}}{d \lambda^{2}}\left[N\left(\sum_{k=0}^{2} \lambda^{k} y_{k}\right)\right]_{\lambda=0} \\
& =y_{2} N^{\prime}\left(y_{0}\right)+\frac{y_{1}^{2}}{2 !} N^{\prime \prime}\left(y_{0}\right),
\end{aligned}
$$

If equations (10) and (11) are substituted into (9), then, we have

$$
\sum_{n=0}^{\infty} y_{n}=\phi+L^{-1} g-L^{-1} \sum_{n=0}^{\infty} A_{n}-L^{-1} P \sum_{n=0}^{\infty} y_{n}
$$

Describing both sides of (12) successively will give

$$
\begin{gathered}
y_{0}=\phi+L^{-1} g \\
y_{1}=-L^{-1} A_{0}-L^{-1} P y_{0} \\
y_{2}=-L^{-1} A_{1}-L^{-1} P y_{1} \\
y_{3}=-L^{-1} A_{2}-L^{-1} P y_{2} \\
\vdots
\end{gathered}
$$

Thus, in general, the recursive relation obtained from the solution is as follows

$$
\begin{gathered}
y_{0}=\phi+L^{-1} g, \\
y_{n+1}=-L^{-1} A_{n}-L^{-1} P y_{n}, n=0,1,2, \ldots,
\end{gathered}
$$

Thus, equation (7) approximate solution becomes:

$$
y \approx \sum_{n=0}^{k} y_{n}, \lim _{k \rightarrow \infty} \sum_{n=0}^{k} y_{n}=y .
$$

\section{Result and Discussion}

In this section, we applied the various numerical methods and definitions discussed above to solve fractional ordinary differential equations (FODE) and report the results as follows.

\subsection{Laplace Decomposition Method}

Consider the following FODE

$$
D_{t}^{\alpha} y(t)=g(t)+N y(t)+P y(t),
$$

with initial condition $\mathrm{y}(0)=\mathrm{c}$, and $D_{t}^{\alpha}$ is the CFD operator with $0<\alpha \leq 1, P$ is a linear operator, $N$ is a nonlinear operator, the function $g$ displays the non-homogeneity of the differential equation, and the function $y$ is said to be determine respect to $t$. Using definition 2.2 and equation (14), we get

$$
y(s)=\frac{y(0)}{s}+\frac{1}{s^{\alpha}} \mathcal{L}[g(t)]+\frac{1}{s^{\alpha}} \mathcal{L}[N y(t)]+\frac{1}{s^{\alpha}} \mathcal{L}[P y(t)] .
$$

Then, applying Laplace transform inverse in (15) will produce

$$
\begin{gathered}
y(t)=y(0)+\mathcal{L}^{-1}\left[\frac{1}{s^{\alpha}} \mathcal{L}[g(t)]\right]+\mathcal{L}^{-1}\left[\frac{1}{s^{\alpha}} \mathcal{L}[N y(t)]\right]+ \\
\mathcal{L}^{-1}\left[\frac{1}{s^{\alpha}} \mathcal{L}[P y(t)]\right] .
\end{gathered}
$$

If we substitute (10) and (11) into (16), we obtain

$$
\begin{gathered}
\sum_{n=0}^{\infty} y_{n}(t)=y(0)+\mathcal{L}^{-1}\left[\frac{1}{s^{\alpha}} \mathcal{L}[g(t)]\right] \\
+\mathcal{L}^{-1}\left[\frac{1}{s^{\alpha}} \mathcal{L}\left[\sum_{n=0}^{\infty} A_{n}\right]\right]+\mathcal{L}^{-1}\left[\frac{1}{s^{\alpha}} \mathcal{L}\left[P \sum_{n=0}^{\infty} y_{n}\right]\right] .
\end{gathered}
$$

Describing both sides of (17) will successively produce

$$
\begin{gathered}
y_{0}=y(0)+\mathcal{L}^{-1}\left[\frac{1}{s^{\alpha}} \mathcal{L}[g(t)]\right], \\
y_{1}=\mathcal{L}^{-1}\left[\frac{1}{s^{\alpha}} \mathcal{L}\left[A_{0}\right]\right]+\mathcal{L}^{-1}\left[\frac{1}{s^{\alpha}} \mathcal{L}\left[P y_{0}\right]\right], \\
y_{2}=\mathcal{L}^{-1}\left[\frac{1}{s^{\alpha}} \mathcal{L}\left[A_{1}\right]\right]+\mathcal{L}^{-1}\left[\frac{1}{s^{\alpha}} \mathcal{L}\left[P y_{1}\right]\right], \\
y_{3}=\mathcal{L}^{-1}\left[\frac{1}{s^{\alpha}} \mathcal{L}\left[A_{2}\right]\right]+\mathcal{L}^{-1}\left[\frac{1}{s^{\alpha}} \mathcal{L}\left[P y_{2}\right]\right], \\
\vdots
\end{gathered}
$$

Thus, in general, the following recursive relation is obtained from the solution of FODE via the Laplace decomposition approach on (14)

$$
\begin{gathered}
y_{0}=y(0)+\mathcal{L}^{-1}\left[\frac{1}{s^{\alpha}} \mathcal{L}[g(t)]\right] \\
y_{n+1}=\mathcal{L}^{-1}\left[\frac{1}{s^{\alpha}} \mathcal{L}\left[A_{n}\right]\right]+\mathcal{L}^{-1}\left[\frac{1}{s^{\alpha}} \mathcal{L}\left[P y_{n}\right]\right], n=0,1,2, \ldots
\end{gathered}
$$

\subsection{Sumudu Decomposition Method}

Consider fractional ordinary differential equation defined in equation (14), Using definition 2.4 and equation (14), we obtain

$$
\mathrm{y}(\mathrm{u})=\mathrm{y}(0)+\mathrm{u}^{\alpha} \mathcal{S}[\mathrm{g}(\mathrm{t})]+\mathrm{u}^{\alpha} \mathcal{S}[\mathrm{Ny}(\mathrm{t})]+\mathrm{u}^{\alpha} \mathcal{S}[\mathrm{Py}(\mathrm{t})]
$$

Also, applying the Sumudu transform inverse in (19) will give

$$
\begin{gathered}
y(t)=y(0)+\mathcal{S}^{-1}\left[u^{\alpha} \mathcal{S}[g(t)]\right]+\mathcal{S}^{-1}\left[u^{\alpha} \mathcal{S}[N y(t)]\right]+ \\
+\mathcal{S}^{-1}\left[u^{\alpha} \mathcal{S}[P y(t)]\right]
\end{gathered}
$$

If we substitute (10) and (11) into (20), we have

$$
\begin{gathered}
\sum_{n=0}^{\infty} y_{n}(t)=y(0)+\mathcal{S}^{-1}\left[u^{\alpha} \mathcal{S}[g(t)]\right]+ \\
+\mathcal{S}^{-1}\left[u^{\alpha} \mathcal{S}\left[\sum_{n=0}^{\infty} A_{n}\right]\right]+\mathcal{S}^{-1}\left[u^{\alpha} \mathcal{S}\left[P \sum_{n=0}^{\infty} y_{n}\right]\right] .
\end{gathered}
$$

Describing both sides of (21) will successively produce

$$
\begin{aligned}
& \mathrm{y}_{0}=\mathrm{y}(0)+\mathcal{S}^{-1}\left[\mathrm{u}^{\alpha} \mathcal{S}[\mathrm{g}(\mathrm{t})]\right], \\
& \mathrm{y}_{1}=\mathcal{S}^{-1}\left[\mathrm{u}^{\alpha} \mathcal{S}\left[\mathrm{A}_{0}\right]\right]+\mathcal{S}^{-1}\left[\mathrm{u}^{\alpha} \mathcal{S}\left[\mathrm{Py}_{0}\right]\right], \\
& \mathrm{y}_{2}=\mathcal{S}^{-1}\left[\mathrm{u}^{\alpha} \mathcal{S}\left[\mathrm{A}_{1}\right]\right]+\mathcal{S}^{-1}\left[\mathrm{u}^{\alpha} \mathcal{S}\left[\mathrm{Py}_{1}\right]\right], \\
& \mathrm{y}_{3}=\mathcal{S}^{-1}\left[\mathrm{u}^{\alpha} \mathcal{S}\left[\mathrm{A}_{2}\right]\right]+\mathcal{S}^{-1}\left[\mathrm{u}^{\alpha} \mathcal{S}\left[\mathrm{Py}_{2}\right]\right],
\end{aligned}
$$

Thus, in general, the following recursive relation is obtained from the solution of FODE via Sumudu decomposition approach on (14) 


$$
\begin{gathered}
y_{0}=y(0)+\mathcal{S}^{-1}\left[u^{\alpha} \mathcal{S}[g(t)]\right], \\
y_{n+1}=\mathcal{S}^{-1}\left[u^{\alpha} \mathcal{S}\left[A_{n}\right]\right]+\mathcal{S}^{-1}\left[u^{\alpha} \mathcal{S}\left[P y_{n}\right]\right], n=0,1,2, \ldots
\end{gathered}
$$

\subsection{Natural Decomposition Method}

Consider fractional ordinary differential equation defined in equation (14), Using definition 2.6 on equation (14), we obtain the solution using the Natural Decomposition method as follows:

$$
\begin{aligned}
y(s, u)=\frac{y(0)}{s} & +\frac{u^{\alpha}}{s^{\alpha}} \mathcal{N}[g(t)]+\frac{u^{\alpha}}{s^{\alpha}} \mathcal{N}[N y(t)]+ \\
& +\frac{u^{\alpha}}{s^{\alpha}} \mathcal{N}[\operatorname{Py}(t)] .
\end{aligned}
$$

Applying the Natural transform inverse in equation (25) will produce

$$
\begin{gathered}
y(t)=y(0)+\mathcal{N}^{-1}\left[\frac{u^{\alpha}}{s^{\alpha}} \mathcal{N}[g(t)]\right] \\
+\mathcal{N}^{-1}\left[\frac{u^{\alpha}}{s^{\alpha}} \mathcal{N}[N y(t)]\right]+\mathcal{N}^{-1}\left[\frac{u^{\alpha}}{s^{\alpha}} \mathcal{N}[P y(t)]\right] .
\end{gathered}
$$

If we substitute (10) and (11) into (24), we will have

$$
\begin{gathered}
\sum_{n=0}^{\infty} y_{n}(t)=y(0)+\mathcal{N}^{-1}\left[\frac{u^{\alpha}}{s^{\alpha}} \mathcal{N}[g(t)]\right] \\
+\mathcal{N}^{-1}\left[\frac{u^{\alpha}}{s^{\alpha}} \mathcal{N}\left[\sum_{n=0}^{\infty} A_{n}\right]\right]+\mathcal{N}^{-1}\left[\frac{u^{\alpha}}{s^{\alpha}} \mathcal{N}\left[P \sum_{n=0}^{\infty} y_{n}\right]\right] .
\end{gathered}
$$

Describing both sides of (25) will successively produce

$$
\begin{aligned}
& y_{0}=y(0)+\mathcal{N}^{-1}\left[\frac{u^{\alpha}}{s^{\alpha}} \mathcal{N}[g(t)]\right], \\
& y_{1}=\mathcal{N}^{-1}\left[\frac{u^{\alpha}}{s^{\alpha}} \mathcal{N}\left[A_{0}\right]\right]+\mathcal{N}^{-1}\left[\frac{u^{\alpha}}{s^{\alpha}} \mathcal{N}\left[P y_{0}\right]\right], \\
& y_{2}=\mathcal{N}^{-1}\left[\frac{u^{\alpha}}{s^{\alpha}} \mathcal{N}\left[A_{1}\right]\right]+\mathcal{N}^{-1}\left[\frac{u^{\alpha}}{s^{\alpha}} \mathcal{N}\left[P y_{1}\right]\right], \\
& y_{3}=\mathcal{N}^{-1}\left[\frac{u^{\alpha}}{s^{\alpha}} \mathcal{N}\left[A_{2}\right]\right]+\mathcal{N}^{-1}\left[\frac{u^{\alpha}}{s^{\alpha}} \mathcal{N}\left[P y_{2}\right]\right],
\end{aligned}
$$

Thus, in general, the following recursive relation is obtained from the solution of FODE via Natural decomposition technique on (14)

$$
\begin{gathered}
y_{0}=y(0)+\mathcal{N}^{-1}\left[\frac{u^{\alpha}}{s^{\alpha}} \mathcal{N}[g(t)],\right. \\
y_{n+1}=\mathcal{N}^{-1}\left[\frac{u^{\alpha}}{s^{\alpha}} \mathcal{N}\left[A_{n}\right]\right]+\mathcal{N}^{-1}\left[\frac{u^{\alpha}}{s^{\alpha}} \mathcal{N}\left[P y_{n}\right]\right], n= \\
0,1,2, \ldots
\end{gathered}
$$

\subsection{Elzaki Decomposition Method}

Consider fractional ordinary differential equation defined in equation (14), Using definition 2.8 on equation (14), we obtain the solution using the Elzaki decomposition approach as follows:

$$
\begin{gathered}
y(v)=v^{2} y(0)+v^{\alpha} \varepsilon[g(t)]+v^{\alpha} \varepsilon[N y(t)]+ \\
+v^{\alpha} \varepsilon[P y(t)] .
\end{gathered}
$$

Applying the Elzaki transform inverse in (27) will give

$$
\begin{gathered}
y(t)=y(0)+\mathcal{E}^{-1}\left[v^{\alpha} \varepsilon[g(t)]\right]+\mathcal{E}^{-1}\left[v^{\alpha} \varepsilon[N y(t)]\right]+ \\
+\mathcal{E}^{-1}\left[v^{\alpha} \varepsilon[P y(t)]\right]
\end{gathered}
$$

Next, we substitute (10) and (11) into (28) to obtain

$$
\begin{gathered}
\sum_{n=0}^{\infty} y_{n}(t)=y(0)+\mathcal{E}^{-1}\left[v^{\alpha} \varepsilon[g(t)]\right]+ \\
+\mathcal{E}^{-1}\left[v^{\alpha} \mathcal{E}\left[\sum_{n=0}^{\infty} A_{n}\right]\right]+\mathcal{E}^{-1}\left[v^{\alpha} \mathcal{E}\left[P \sum_{n=0}^{\infty} y_{n}\right]\right] .
\end{gathered}
$$

By describing both sides of (29) will successively produce

$$
\begin{aligned}
& \mathrm{y}_{0}=\mathrm{y}(0)+\mathcal{E}^{-1}\left[\mathrm{v}^{\alpha} \mathcal{E}[\mathrm{g}(\mathrm{t})]\right], \\
& \mathrm{y}_{1}=\mathcal{E}^{-1}\left[\mathrm{v}^{\alpha} \mathcal{E}\left[\mathrm{A}_{0}\right]\right]+\mathcal{E}^{-1}\left[\mathrm{v}^{\alpha} \mathcal{E}\left[\mathrm{Py}_{0}\right]\right], \\
& \mathrm{y}_{2}=\mathcal{E}^{-1}\left[\mathrm{v}^{\alpha} \mathcal{E}\left[\mathrm{A}_{1}\right]\right]+\mathcal{E}^{-1}\left[\mathrm{v}^{\alpha} \mathcal{E}\left[\mathrm{Py}_{1}\right]\right], \\
& \mathrm{y}_{3}=\mathcal{E}^{-1}\left[\mathrm{v}^{\alpha} \mathcal{E}\left[\mathrm{A}_{2}\right]\right]+\mathcal{E}^{-1}\left[\mathrm{v}^{\alpha} \mathcal{E}\left[\mathrm{Py}_{2}\right]\right],
\end{aligned}
$$

Hence, in general, the following recursive relation is obtained from the solution of FODE via Elzaki decomposition technique on (14)

$$
\begin{gathered}
y_{0}=y(0)+\mathcal{E}^{-1}\left[v^{\alpha} \mathcal{E}[g(t)]\right], \\
y_{n+1}=\mathcal{E}^{-1}\left[v^{\alpha} \varepsilon\left[A_{n}\right]\right]+\mathcal{E}^{-1}\left[v^{\alpha} \mathcal{E}\left[P y_{n}\right]\right], n=0,1,2, \ldots
\end{gathered}
$$

\subsection{Mohand Decomposition Method}

Consider fractional ordinary differential equation defined in equation (14), Using Mohand transform on (14) and based on definition 2.10, we have

$$
\begin{gathered}
y(v)=v y(0)+\frac{1}{v^{\alpha}} \mathcal{M}[g(t)]+\frac{1}{v^{\alpha}} \mathcal{M}[N y(t)]+ \\
+\frac{1}{v^{\alpha}} \mathcal{M}[\operatorname{Py}(t)] .
\end{gathered}
$$

Applying the Mohand transform inverse in (31) will produce

$$
\begin{gathered}
y(t)=y(0)+\mathcal{M}^{-1}\left[\frac{1}{v^{\alpha}} \mathcal{M}[g(t)]\right] \\
+\mathcal{M}^{-1}\left[\frac{1}{v^{\alpha}} \mathcal{M}[N y(t)]\right]+\mathcal{M}^{-1}\left[\frac{1}{v^{\alpha}} \mathcal{M}[P y(t)]\right] .
\end{gathered}
$$

Next is substituting (10) and (11) into (32) to obtain

$$
\begin{gathered}
\sum_{n=0}^{\infty} y_{n}(t)=y(0)+\mathcal{M}^{-1}\left[\frac{1}{v^{\alpha}} \mathcal{M}[g(t)]\right] \\
+\mathcal{M}^{-1}\left[\frac{1}{v^{\alpha}} \mathcal{M}\left[\sum_{n=0}^{\infty} A_{n}\right]\right]+ \\
+\mathcal{M}^{-1}\left[\frac{1}{v^{\alpha}} \mathcal{M}\left[P \sum_{n=0}^{\infty} y_{n}\right]\right] .
\end{gathered}
$$


Describing both sides of equation (33) will successively produce

$$
\begin{aligned}
\mathrm{y}_{0} & =\mathrm{y}(0)+\mathcal{M}^{-1}\left[\frac{1}{\mathrm{v}^{\alpha}} \mathcal{M}[\mathrm{g}(\mathrm{t})]\right], \\
\mathrm{y}_{1} & =\mathcal{M}^{-1}\left[\frac{1}{\mathrm{v}^{\alpha}} \mathcal{M}\left[\mathrm{A}_{0}\right]\right]+\mathcal{M}^{-1}\left[\frac{1}{\mathrm{v}^{\alpha}} \mathcal{M}\left[\mathrm{Py}_{0}\right]\right], \\
\mathrm{y}_{2} & =\mathcal{M}^{-1}\left[\frac{1}{\mathrm{v}^{\alpha}} \mathcal{M}\left[\mathrm{A}_{1}\right]\right]+\mathcal{M}^{-1}\left[\frac{1}{\mathrm{v}^{\alpha}} \mathcal{M}\left[\mathrm{Py}_{1}\right]\right], \\
\mathrm{y}_{3} & =\mathcal{M}^{-1}\left[\frac{1}{\mathrm{v}^{\alpha}} \mathcal{M}\left[\mathrm{A}_{2}\right]\right]+\mathcal{M}^{-1}\left[\frac{1}{\mathrm{v}^{\alpha}} \mathcal{M}\left[\mathrm{Py}_{2}\right]\right], \\
& \vdots
\end{aligned}
$$

Therefore, in general, the following recursive relation is obtained from the solution of FODE via Mohand decomposition method (14)

$$
\begin{gathered}
y_{0}=y(0)+\mathcal{M}^{-1}\left[\frac{1}{v^{\alpha}} \mathcal{M}[g(t)]\right], \\
y_{n+1}=\mathcal{M}^{-1}\left[\frac{1}{v^{\alpha}} \mathcal{M}\left[A_{n}\right]\right]+\mathcal{M}^{-1}\left[\frac{1}{v^{\alpha}} \mathcal{M}\left[P y_{n}\right]\right], \\
n=0,1,2, \ldots
\end{gathered}
$$

\subsection{Kashuri-Fundo Decomposition Method}

Consider fractional ordinary differential equation defined in equation (14), Using Kashuri-Fundo decomposition method (14) and based on definition 2.12, we have

$$
\begin{aligned}
y(v)=v y(0) & +v^{2 \alpha} \mathcal{K}[g(t)]+v^{2 \alpha} \mathcal{K}[N y(t)]+ \\
& +v^{2 \alpha} \mathcal{K}[P y(t)] .
\end{aligned}
$$

Applying the Kashuri-Fundo transform inverse in (35) will give

$$
\begin{gathered}
y(t)=y(0)+\mathcal{K}^{-1}\left[v^{2 \alpha} \mathcal{K}[g(t)]\right] \\
+\mathcal{K}^{-1}\left[v^{2 \alpha} \mathcal{K}[N y(t)]\right]+\mathcal{K}^{-1}\left[v^{2 \alpha} \mathcal{K}[P y(t)]\right] .
\end{gathered}
$$

If we substitute (10) and (11) into (36), we have

$$
\begin{gathered}
\sum_{n=0}^{\infty} y_{n}(t)=y(0)+\mathcal{K}^{-1}\left[v^{2 \alpha} \mathcal{K}[g(t)]\right]+ \\
+\mathcal{K}^{-1}\left[v^{2 \alpha} \mathcal{K}\left[\sum_{n=0}^{\infty} A_{n}\right]\right]+\mathcal{K}^{-1}\left[v^{2 \alpha} \mathcal{K}\left[P \sum_{n=0}^{\infty} y_{n}\right]\right] .
\end{gathered}
$$

Describing both sides of (37) will successively produce

$$
\begin{aligned}
\mathrm{y}_{0} & =\mathrm{y}(0)+\mathcal{K}^{-1}\left[\mathrm{v}^{2 \alpha} \mathcal{K}[\mathrm{g}(\mathrm{t})]\right], \\
\mathrm{y}_{1} & =\mathcal{K}^{-1}\left[\mathrm{v}^{2 \alpha} \mathcal{K}\left[\mathrm{A}_{0}\right]\right]+\mathcal{K}^{-1}\left[\mathrm{v}^{2 \alpha} \mathcal{K}\left[\mathrm{Py}_{0}\right]\right] \\
\mathrm{y}_{2} & =\mathcal{K}^{-1}\left[\mathrm{v}^{2 \alpha} \mathcal{K}\left[\mathrm{A}_{1}\right]\right]+\mathcal{K}^{-1}\left[\mathrm{v}^{2 \alpha} \mathcal{K}\left[\mathrm{Py}_{1}\right]\right] \\
\mathrm{y}_{3} & =\mathcal{K}^{-1}\left[\mathrm{v}^{2 \alpha} \mathcal{K}\left[\mathrm{A}_{2}\right]\right]+\mathcal{K}^{-1}\left[\mathrm{v}^{2 \alpha} \mathcal{K}\left[\mathrm{Py}_{2}\right]\right]
\end{aligned}
$$

Therefore, in general, the following recursive relation is obtained from the solution of FODE using the Kashuri-Fundo decomposition method on (14)

$$
\begin{gathered}
y_{0}=y(0)+\mathcal{K}^{-1}\left[v^{2 \alpha} \mathcal{K}[g(t)]\right] \\
y_{n+1}=\mathcal{K}^{-1}\left[v^{2 \alpha} \mathcal{K}\left[A_{n}\right]\right]+\mathcal{K}^{-1}\left[v^{2 \alpha} \mathcal{K}\left[P y_{n}\right]\right], \\
n=0,1,2, \ldots
\end{gathered}
$$

\section{Conclusions}

This paper is structured to combine the Adomian decomposition method with various integral transforms, including Natural, Sumudu, Laplace, Elzaki, Mohand, and Kashuri-Fundo. The main finding of our study shows that the combine numerical methods considered produce excellent numerical performance for solving fractional ordinary differential equations. For future evaluations, the results of this study can be applied to various disciplines. Such as communication security systems and electronic circuits.

\section{Acknowledgements}

The authors are grateful to Universitas Padjadjaran through the Directorate of Research, Community Service and Innovation for providing the publication costs.

\section{Conflict of Interest}

There is no conflict of interest as declare by the authors.

\section{REFERENCES}

[1] G. Adomian, A Review of the Decomposition Method in Applied Mathematics, Journal of Mathematical Analysis and Applications, 1988, Vol. 135, No. 2, 501-544.

[2] S. Mobayen, A. Fekih, S. Vaidyanathan, A. Sambas. Chameleon Chaotic Systems with Quadratic Nonlinearities: An Adaptive Finite-Time Sliding Mode Control Approach and Circuit Simulation. IEEE Access, 2021, Vol. 9, 64558-64573.

[3] J. Duan, R. Rach, D. Baleanu, A. Wazwaz, A review of the Adomian decomposition method and its applications to fractional differential equations, Commun. Frac. Calc, 2012., Vol. 3, No. 2, 73-99.

[4] A. Sambas, S. Vaidyanathan, S. Zhang, Y. Zeng, M. A. Mohamed, M. Mamat. A new double-wing chaotic system with coexisting attractors and line equilibrium: bifurcation analysis and electronic circuit simulation. IEEE Access, 2019, Vol. 7, 115454-115462.

[5] M. P. Goswami, N. Jha, Triple Laplace Transform in Bicomplex Space with Application, Mathematics and Statistics, Vol. 8, No. 4, 443-450, 2020. DOI: 10.13189/ms.2020.080411. 
[6] F. Shakeri, M. Dehghan, Numerical solution of a biological population model using He's variational iteration method. Computers \& Mathematics with applications, 2007, Vol. 54, No. 7-8, 1197-1209.

[7] M. Farman, M. U. Saleem, A. Ahmad, M. O. Ahmad, Analysis and numerical solution of SEIR epidemic model of measles with non-integer time fractional derivatives by using Laplace Adomian Decomposition Method. Ain Shams Engineering Journal, 2018, Vol. 9, No. 4, 3391-3397.

[8] G. Asadi-Cordshooli, A. Vahidi, R. Norouzi, Solution of Inverse Kinematic Problem of a 2DOF Robot Using Decomposition Method. Journal of New Researches in Mathematics, 2015, Vol. 1, No. 1, 5-13.

[9] S. Vaidyanathan, A. Sambas, M. Mamat, W. S. M. Sanjaya, A new three-dimensional chaotic system with a hidden attractor, circuit design and application in wireless mobile robot. Archives of Control Sciences, 2017, Vol. 27, No. 4 , 541-554.

[10] Sukono, A. Sambas, S. He, H. Liu, S. Vaidyanathan, Y. Hidayat, J. Saputra, Dynamical analysis and adaptive fuzzy control for the fractional-order financial risk chaotic system. Advances in Difference Equations, 2020, Vol. 674, No. 1, $1-12$.

[11] N. Manzoor, K. Maqbool, O. A. Bég, S. Shaheen, Adomian decomposition solution for propulsion of dissipative magnetic Jeffrey biofluid in a ciliated channel containing a porous medium with forced convection heat transfer. Heat Transfer-Asian Research, 2019, Vol. 48, No. 2, 556-581.

[12] S. Vaidyanathan, A. Sambas, B. Abd-El-Atty, A. A. Abd El-Latif, E. Tlelo-Cuautle, O. Guillén-Fernández, M. Mamat, M. A. Mohamed, M. Alcin. M. Tuna, I. Pehlivan, I. Koyuncu, M. A. H. Ibrahim, A 5-D multi-stable hyperchaotic two-disk dynamo system with no equilibrium point: Circuit design, FPGA realization and applications to TRNGs and image encryption. IEEE Access, 2021, Vol. 9, 81352-81369.

[13] N. A. A. Fataf, M. A. Rahim, S. He, S. Banerjee, A Communication Scheme based on Fractional Order Chaotic Laser for Internet of Things. Internet of Things, 2021, Vol. $15,100425$.

[14] A. Sambas, S. Vaidyanathan, S. Zhang, W. T. Putra, M. Mamat, M. A. Mohamed, Multistability in a Novel Chaotic System with Perpendicular Lines of Equilibrium: Analysis, Adaptive Synchronization and Circuit Design. Engineering Letters, 2019, Vol. 27, No. 4, 744-751.

[15] J. H. Kuang, C. J. Chen, Adomian decomposition method used for solving nonlinear pull-in behavior in electrostatic micro-actuators. Mathematical and computer modelling, 2005, Vol. 41, No. 13, 1479-1491.

[16] J. J. Parra, Adomian Decomposition of the Flowfield in a Simulated Rocket Motor, Master Thesis, The University of Tennessee, Knoxville, 2014.

[17] S. Vaidyanathan, E. Tlelo-Cuautle, A. Sambas, L. G. Dolvis, O. Guillén-Fernández, FPGA design and circuit implementation of a new four-dimensional multistable hyperchaotic system with coexisting attractors. International Journal of Computer Applications in Technology, 2020, Vol. 64, No. 3, 223-234.
[18] J. B. Yindoula, P. Youssouf, G. Bissanga, F. Bassono, B. Some. Application of the Adomian decomposition method and Laplace transform method to solving the convection diffusion-dissipation equation. International Journal of Applied Mathematics Research, 2014, Vol. 3, No. 1, 30-36.

[19] B. Zhen, Z. Song. The Study for Synchronization between Two Coupled FitzHugh-Nagumo Neurons Based on the Laplace Transform and the Adomian Decomposition Method. Neural Plasticity, 2021, Vol. 2021, 6657835.

[20] F. Chen, Q. Q. Liu. Adomian Decomposition Method Combined with Padé Approximation and Laplace Transform for Solving a Model of HIV Infection of CD4+ T Cells. Discrete Dynamics in Nature and Society, 2015, Vol. 2015, 584787.

[21] N. Doğan. Solution of the System of Ordinary Differential Equations by Combined Laplace Transform-Adomian Decomposition Method. Mathematical and Computational Applications, 2012, Vol. 17, No. 3, 203-211.

[22] N. B. Manjare, H. T. Dinde. Sumudu decomposition method for solving fractional Bratu-type differential equations. Journal of Scientific Research, 2020, Vol. 12, No. 4, 585-605.

[23] H. Eltayeb, A. Kılıçman, S. Mesloub. Application of Sumudu decomposition method to solve nonlinear system Volterra integrodifferential equations. In Abstract and Applied Analysis, 2014, Vol. 2014, 503141.

[24] M.A Ramadan, M. A. S. Al-Luhaibi. Application of sumudu decomposition method for solving linear and nonlinear Klein-Gordon equations. Int. J. Soft Comput. Eng, 2014, 3(6), 138-141.

[25] H. Khan, C. Tunç, R. A. Khan, A. G. Shirzoi, A. Khan. Approximate Analytical Solutions of Space-Fractional Telegraph Equations by Sumudu Adomian Decomposition Method. Applications \& Applied Mathematics, 2018, Vol. 13, No. 2, 781-802.

[26] D. Kumar, J. Singh, A. Kılıçman. An efficient approach for fractional Harry Dym equation by using Sumudu transform. In Abstract and Applied Analysis, 2013, Vol. 2013, 608943.

[27] H. Z. Mjthap, S. N. Al-Azzawi. Mixing Sumudu transform and Adomain decomposition method for solving Riccati equation of variable fractional order. Journal of Interdisciplinary Mathematics, 2019, Vol. 22, No. 8, 1559-1563.

[28] Alfaqeih, S., \& Misırlı, E. (2021). On Convergence Analysis and Analytical Solutions of the Conformable Fractional Fitzhugh-Nagumo Model Using the Conformable Sumudu Decomposition Method. Symmetry, 13(2), 243.

[29] P. Veeresha, D. G. Prakasha, J. Singh. Solution for fractional forced $\mathrm{KdV}$ equation using fractional natural decomposition method. AIMS Mathematics, 2020, Vol. 5, No. 2, 798-810.

[30] H. Khan, R. Shah, D. Baleanu, P. Kumam, M. Arif. Analytical solution of fractional-order hyperbolic telegraph equation, using natural transform decomposition method. Electronics, 2019, Vol. 8, No. 9, 1015.

[31] R. Shah, H. Khan, S. Mustafa, P. Kumam, M. Arif. 
Analytical solutions of fractional-order diffusion equations by natural transform decomposition method. Entropy, 2019, Vol. 21, No. 6, 557.

[32] R. I. Nuruddeen. Elzaki decomposition method and its applications in solving linear and nonlinear Schrodinger equations. Sohag Journal of Mathematics, 2017, Vol. 4, No. 2, $1-5$.

[33] A. C. Varsoliwala, T. R. Singh. Mathematical modelling of atmospheric internal waves phenomenon and its solution by Elzaki Adomian Decomposition Method. Journal of Ocean Engineering and Science, 2021, In Press.

[34] H. Khan, A. Khan, P. Kumam, D. Baleanu, M. Arif. An approximate analytical solution of the Navier-Stokes equations within Caputo operator and Elzaki transform decomposition method. Advances in Difference Equations, 2020, Vol. 662, No. 1, 1-23.

[35] R. Shah, U. Farooq, H. Khan, D. Baleanu, P. Kumam, M. Arif, Fractional View Analysis of Third Order Kortewege-De Vries Equations, Using a New Analytical Technique, Front. Phys., 2020, Vol. 7, 11 pages.

[36] I. Ali, H. Khan, U. Farooq, D. Baleanu, P. Kumam, M. Arif, An Approximate-Analytical Solution to Analyze Fractional View of Telegraph Equations, IEEE Access, 2020, Vol. 8, 25638-25649.

[37] A. Kashuri, A. Fundo, A New Integral Transform, Advances in Theoretical and Applied Mathematics, 2013, Vol. 8, No. 1, 27-43.
[38] I. Sumiati, Sukono A. T. Bon, Adomian Decomposition Method and The New Integral Transform, Proceedings of the 2nd African International Conference on Industrial Engineering and Operations Management, Harare, Zimbabwe, December, 2020, 1882-1887.

[39] J. L. Schiff, The Laplace Transform: Theory and Applications, New York: Springer, 1999.

[40] I. Podlubny, Fractional Differential Equations, California: Academic Press, 1999.

[41] F. M. Belgacem, A. A. Karaballi, Sumudu Transform Fundamental Properties Investigations and Applications, Journal of Applied Mathematics and Stochastic Analysis, 2006, 1-23.

[42] V. B. L. Chaurasia, J. Singh, Application of Sumudu Transform in Schödinger Equation Occurring in Quantum Mechanics, Applied Mathematical Sciences, 2010, vol. 4, No. 57-60, 2843-2850.

[43] F. B. M. Belgacem, R. Silambrasan, Advances in the Natural transform. 9th International Conference on Mathematical Problems in Engineering, Aerospace and Sciences AIP Conf. Proc., 2012, vol. 1493, No. 1, 106-110.

[44] T. M. Elzaki, The New Integral Transform "ELzaki Transform", Global Journal of Pure and Applied Mathematics, 2011, Vol. 7, No. 1, 57-64.

[45] M. M. A. Mahgoub, The New Integral Transform "Mohand Transform", Advances in Theoretical and Applied Mathematics, 2017, Vol. 12, No. 2, 113-120. 